\title{
Una mirada a los valores en el contexto universitario: Cuba
}

\author{
A Glimpse into Values in University Contexts: Cuba \\ Um olhar sobre os valores no contexto universitário: Cuba
}

\author{
Edelmis Cristina Reyes-Quiñones \\ Centro de Referencia de Educación de Avanzada (CREA) \\ Universidad Tecnológica de La Habana, Cujae \\ Cuba
}

Recibido: 19/02/ 2020 - Aceptado: 20/03/2020

\begin{abstract}
Resumen
Los valores en el contexto universitario son intencionalmente priorizados en todos los espacios sociales, por tanto la credibilidad y la pertinencia de la universidad del siglo XXI se harán evidentes en la medida en que se forjen profesionales que sean hombres y mujeres de paz y de decoro, cuya sólida formación humanista y alta capacitación científico-técnica sean fuente de inspiración para acometer con audacia responsable, la búsqueda de soluciones que permitan salvar al planeta y construir un mundo mejor para todas las personas.
\end{abstract}

Palabras claves: Valores, universidad, profesionalidad, educación, humanismo, Temas de Nuestra América.

Abstract

The values in the university context, is intentionally prioritized in all social spaces, therefore the credibility and relevance of the university of the XXI century will be evident to the extent that professionals are forged that are men and women of peace and propriety, whose solid humanistic formation and high scientific-technical training are a source of inspira-

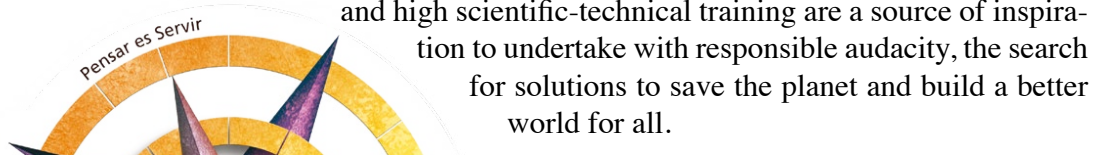

Keywords: values, university, profesvalores, universidad, profesionalidad, educación, humanismo, Nuestra America [Our America] 


\section{Resumo}

Os valores no contexto universitário são intencionalmente priorizados em todos os espaços sociais, portanto a credibilidade e relevância da universidade do século XXI será evidente na medida em que forem forjados profissionais que sejam homens e mulheres de paz e decoro, cuja sólida formação humanística e alta formação técnico-científica são uma fonte de inspiração para empreender com audácia responsável, a busca de soluções que salvem o planeta e construam um mundo melhor para todos.

Palavras chave: valores, universidade, profissionalismo, educação, humanismo, tópicos de nossa américa

\section{Introducción}

Según Robbins (1999), los valores representan convicciones básicas de que un modo específico de conducta es preferible, desde el punto de vista personal o social, a un modo contrario de comportamiento. Constituyen los cimientos de la comprensión de las actitudes, la motivación y la conducta, además de que influyen en la percepción.

Por su parte, Díaz Llorca (2010) plantea que son un accionar sistemático que marcan nuestras acciones y que están condicionados por nuestra forma de pensar, por nuestras estructuras de pensamientos, por los aspectos que nos enseñaron y por las experiencias que vamos adquiriendo, es decir, por nuestras creencias. Creencias y valores marchan juntos, no es posible separarlos.

Todo valor posee un antivalor y florecen aquellos que nos mantienen en nuestra zona de confort, determina las acciones que consideramos justas y aceptables, según nuestras creencias y nos otorga un lugar en el contexto social. Las sociedades actúan y se desarrollan inmersas en el contexto económico-político en que viven, basadas en sus propias experiencias y los conocimientos adquiridos, determinan su cultura, la que se va trasformando con su propio desarrollo. Por tanto, las sociedades actúan según sus creencias, según sus percepciones de la realidad.

Los cambios ocurridos al triunfo de la Revolución provocaron un vuelco en la vida del pueblo cubano, se experimentaron transformaciones sociales profundas que implicaron nuevas formas de actuación, dadas las posibilidades de vida que se abrían para la gran mayoría. Esto trajo aparejada una metamorfosis cultural, matizada por la participación en la vida del país de grupos hasta ese entonces marginados, la apertura de posibilidades educacionales y laborales antes inaccesibles

52 Una mirada a los valores en el contexto universitario: Cuba Edelmis Cristina Reyes-Quiñones

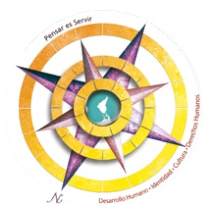


y una nueva manera de convivencia, desprovista de exclusiones como el racismo y la desigualdad de género. Surgía una nueva nación que, en el decurso del tiempo, luchaba por mantener y consolidar su cultura.

La situación política económica y social que se vivía en los años noventa era crítica, producto del período especial y del recrudecimiento del bloqueo económico, el desigual intercambio comercial y de influencias, la existencia de un mundo unipolar, la globalización del capitalismo bajo los fundamentos ideológicos del neo liberalismo y la hegemonía del imperialismo yanqui como gendarme.

Fidel Castro Ruz caracterizó el momento que se vivía:

... no es un mundo lleno de bondad, es un mundo lleno de egoísmo; no es un mundo lleno de justicia, es un mundo lleno de explotación, de abuso, de saqueo, donde un número de millones de niños mueren cada año $-y$ podrían salvarse - simplemente porque les faltan unos centavos de medicamentos, un poco de vitaminas y sales minerales y unos pocos dólares de alimentos, suficientes para que puedan vivir ... (Castro, 2005, s/p)

La dirección de la Revolución, para reanimar la economía y aliviar la crisis, traza la estrategia al circunscribir medidas como despenalizar la tenencia y el empleo de la moneda libremente convertible, estimular la inversión extranjera, crear mercados agropecuarios e industriales y ampliar el trabajo por cuenta propia, entre otras, con un impacto en la sociedad, evaluado por el comandante en jefe, cuando declaraba

... estos acontecimientos influyeron ideológicamente, hubo mucha gente que se confundió en los primeros momentos de aquel proceso. ... También influyeron ideológicamente los desastres, la increíble evolución de los acontecimientos afectó la confianza, el ánimo, la conciencia de mucha gente ... (Castro, 1991 ,s/p)

Muchas de ellas provocaron desigualdades en la equidad social y la moralidad alcanzada por la sociedad cubana hasta ese momento y motivaron que, en algunos sectores vulnerables de la población, resurgieran males sociales, prácticamente erradicados por la Revolución, como el consumismo, el egoísmo, la indisciplina social, la desestimulación ante el trabajo, la pérdida de la significación y valor del trabajo, la búsqueda de dinero o de una vida suntuosa por vías fáciles o ilegales, la aparición de una nueva modalidad de la prostitución, la falta de ejemplaridad y honestidad por 
sectores del funcionariado estatal, el mercado negro y otras manifestaciones de corrupción y delito.

En este contexto, se orienta defender la independencia, la justicia social y la cultura nacional, intensificando el estudio y la divulgación de las mejores tradiciones de la historia, del pensamiento político y de la cultura; con énfasis en la obra de José Martí, Ernesto Guevara y Fidel Castro. Es aprobada en diciembre de 1996 la Ley de Reafirmación de la Dignidad y Soberanías Cubanas por la Asamblea Nacional; es proclamada en marzo de 1997 la Declaración de los Mambises del Siglo XX; en 1999 el Juramento de Baraguá, entre otras leyes y declaraciones que expresaban la decisión del pueblo de resistir $\mathrm{y}$ vencer frente al acoso imperialista.

La formación de valores no es espontánea. Los valores, en su condicionamiento sociohistórico, su conceptualización y jerarquización, varían en dependencia de múltiples factores: la familia, la escuela, la comunidad, la ideología imperante en la sociedad, la situación económico - social, entre otros. De ahí que, en los centros de enseñanza, sea una labor intencionalmente priorizada y a los centros de educación superior (CES) les corresponde un papel importante en ese proceso de formación de valores.
Los valores que identifican a la sociedad socialista son la dignidad, el patriotismo, el humanismo, la solidaridad, la responsabilidad, la laboriosidad, la honradez, la honestidad y la justicia, son aspiraciones de la Política Educacional Cubana fomentar un modelo de persona éticamente educada y socialmente profesional, capaz de enfrentar, de manera reflexiva y creadora, los enormes retos de los tiempos actuales.

Miguel Díaz-Canel Bermúdez, en su intervención en el VII Congreso Internacional Universidad 2010, expresó:

La credibilidad y la pertinencia de la universidad del siglo XXI se harán evidentes en la medida en que se forjen profesionales que sean hombres y mujeres de paz y de decoro, cuya sólida formación humanista y alta capacitación científico-técnica sean fuente de inspiración para acometer con audacia responsable, la búsqueda de soluciones que permitan salvar al planeta y construir un mundo mejor para todos (Díaz-Canel, 2010).

En octubre de 2006 se aprueba el Programa Director para el Reforzamiento de los Valores Fundamentales en la Sociedad Cubana actual, el que se actualiza en el 2012 como Programa Director para la Educación en el Sistema de Valores de la

54 Una mirada a los valores en el contexto universitario: Cuba Edelmis Cristina Reyes-Quiñones 
Revolución Cubana, posteriormente como Programa Director.

Este Programa tiene como objetivo contribuir, mediante su aplicación práctica, a reforzar la formación de valores, tales como la dignidad, el patriotismo, el humanismo, la solidaridad, la responsabilidad, la laboriosidad, la honradez, la honestidad y la justicia.

En los planes de estudio de las universidades el componente humanista está estrechamente vinculado al aspecto de la ética y a la formación de una moral profesional, en correspondencia con las exigencias del país.

En el curso 2011-2012 el Instituto Superior Politécnico José Antonio Echeverría (ISPJAE-Cujae), actualmente Universidad Tecnológica de La Habana José Antonio Echeverría (UTH-Cujae), elabora, por primera vez, las estrategias y objetivos de profesionales en ingeniería (CUJAE, 2011). El material refiere al estudiantado, los retos y oportunidades a los que se enfrentará en un mundo regido por la globalización y la sociedad del conocimiento. Se enfatiza en el compromiso con un desarrollo sustentable, con el cuidado del medio ambiente y el bienestar del ser humano.

En general, los ingenieros y las ingenieras son líderes de proyectos, crean sistemas, conducen y guían obras. Por ello, la sistematización de cada uno de los procesos que llevará a cabo, mediante la toma de decisiones, es necesaria, para la formación de profesionales capaces de contribuir a la implementación de los Lineamientos de la Política Económica y Social de la Revolución aprobados en el VII Congreso del Partido (CC PCC, 2017).

El auge en el siglo XXI del uso de las tecnologías de la información $\mathrm{y}$ las comunicaciones (TIC) en el proceso de enseñanza-aprendizaje ha creado nuevas pautas en la adquisición del conocimiento, posibilitado por la consulta inmediata a potentes bases de datos de información, uso de modelos teóricos simulados, posibilidad de acceso a bibliografías de disímiles autorías, almacenamiento y consulta de información, análisis financieros. Esto implica que el estudiantado necesita una mejor formación profesional que le permita orientarse, de modo autónomo, en ese cúmulo de información y, críticamente, utilizarla de forma adecuada en su vinculación universidad-empresa.

La ingeniería, profesión eminentemente creativa y dinámica, pone al servicio del ser humano métodos y procedimientos para usar y 
aprovechar, con la máxima eficacia, los materiales y fuerzas que la naturaleza le brinda para satisfacer las necesidades sociales, donde resulta primordial seguir trabajando nuevas formas y métodos, nuevos diseños e integraciones, que en el campo de la educación de la personalidad propicien un desarrollo moral que actúe regulando el comportamiento ético revolucionario, culto e integral de profesionales a quienes se aspira, como elemento transformador de la sociedad cubana actual.

El ingeniero o ingeniera realiza sus actividades dentro de un marco de ética profesional, por lo cual es necesario que, desde los inicios de sus estudios, reciba orientaciones psicológicas, sociológicas, pedagógicas, morales, educacionales, económicas y sociales en general, vinculadas íntimamente con la familia, la comunidad, la escuela, el centro laboral, la sociedad en su conjunto y el Estado.

Para cumplir el encargo social de la educación, se necesita trabajar en el desarrollo de las habilidades profesionales, de los valores de la profesión acordes con el progreso científico-técnico, por el mejoramiento del sistema de influencias educativas, por elevar la calidad del proceso de enseñanza en la educación superior, temáticas debatidas en los
Congresos Universidad, 2018 y en Pedagogía 2019.

La ingeniería industrial es una de las ramas del conocimiento que involucra la creatividad y la puesta en práctica de los principios de la ciencia a los procesos y procedimientos de la industria. Está relacionada con el diseño e instalación y mejoramiento operacional de sistemas integrados de recursos humanos, materiales $\mathrm{y}$ equipos, tiene conocimientos de la física, la matemática, la química y las ciencias sociales, para dirigir, analizar, organizar y evaluar el proceso de funcionamiento de la industria.

El proyecto El proceso de enseñanza-aprendizaje de carreras de ciencias técnicas de la Educación Superior en ambientes con alta presencia de las tecnologías de la información y las comunicaciones, dirigido por la Dra. C. Raquel Bermúdez Morris, se centra en el análisis del papel que representan los avances tecnológicos en los procesos de enseñanza-aprendizaje y está vinculado al Programa Nacional Problemas actuales del sistema educativo cubano. Perspectivas de desarrollo.

La educación en la profesionalidad del futuro ingeniero e ingeniera debe, ante todo, influir en la concientización del ejercicio de la profesión

56 Una mirada a los valores en el contexto universitario: Cuba Edelmis Cristina Reyes-Quiñones

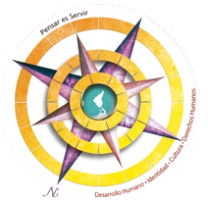


con la mayor eficiencia y eficacia posible, el respeto a la profesión, basar sus principios en las leyes y regularidades de la ciencia, ganarse su reputación con el resultado de su trabajo y su impacto en la sociedad, aspirar a los estándares superiores de su disciplina, mostrar compromiso con su país al brindar el mejor servicio, actuar con decoro manteniendo en todo momento el respeto hacia sus colegas profesionales, para la toma de decisiones, los reglamentos y las leyes vigentes, todo lo cual se resume en el valor profesionalidad.

En el marco teórico que ofrece la axiología marxista leninista y las ciencias pedagógicas en lo referido a la didáctica y a los enfoques metodológicos sobre el proceso de profesionalización y la formación de valores, el estudio de documentos normativos para la labor educativa han resultado de vital importancia para desarrollar esta línea de trabajo científico. Un valioso aporte a la comprensión de la formación axiológica de las generaciones jóvenes la brinda el enfoque histórico cultural de L. S. Vigotsky y sus seguidores.

La universidad cubana actual como institución social está enfrascada en ratificar su modelo de universidad científica, tecnológica y humanista, de universidad dinámica y práctica, caracterizada por la formación de valores y dedicada a la creación, promoción y difusión del conocimiento, el desarrollo de la ciencia y la innovación tecnológica, que asume la responsabilidad de poner su formación al servicio de las demandas y necesidades del desarrollo social. (Díaz-Canel, 2011, p.10)

Esta no se estructura solo para dar respuesta a las necesidades del mercado laboral, sino que su perfil se modela en la formación de profesionales con integridad, que centren su atención fundamentalmente en el desarrollo de su personalidad (sencillez, modestia, altruismo, responsabilidad, fidelidad, sinceridad, honradez, o virtudes como la prudencia, la justicia, la esperanza) y se desempeñen con éxito, por lo que constituye un aspecto significativo de su formación básica, la conciencia y el compromiso social como prioridad principal.

El objetivo para la formación de los profesionales es consecuente con la concepción expresada de: "lograr profesionales cultos, competitivos, altamente eficientes y preparados para servir a la patria, mediante un sistema que garantice su formación integral, correspondiendo una sólida preparación científico-técnica, humanista y de altos valores" (Horruitiner, 2006, p. 27). La función básica, formar profesionales útiles a 
la sociedad, sustentada en el precepto martiano "... Puesto que a vivir viene el hombre, la educación ha de prepararlo para la vida". (Martí, 1990, p. 11).

En la bibliografía acerca del tema de la profesionalidad se incorpora, junto a los conceptos de perfeccionamiento, actualización y superación, el principio de la educación permanente y continua, condicionada por sus necesidades individuales, sociales y del desarrollo científico-tecnológico actual y futuro.

La educación permanente se constituye en una integración de la formación inicial y continua en la formación profesional “... que se remite al trabajo como eje del proceso educativo, fuente de conocimiento y objeto de transformación, que privilegia la participación colectiva multidisciplinaria, que favorece la construcción dinámica de nuevos conocimientos a través de la investigación, el manejo analítico de la información y el intercambio de saberes y experiencias" (Roschke; Jadead, 2010, p. 7).

Esta noción precisa la necesidad de convertir al sujeto profesional en un agente activo, que parte de una instrucción formalizada para ocupar un empleo dentro de una profesión y que debe actualizarse permanentemente en sus conocimientos, prácticas y actitudes con profesionalidad creciente. La profesionalidad no solo privilegia el área instrumental, sino que enfatiza en dominios sobre la profesión, la cosmovisión, la ética, el sentido de pertenencia y la identidad profesional, elementos esenciales en la formación humanista declarados como premisas de la formación inicial profesional por el Ministerio de Educación Superior (MES).

Sobre los valores morales y su formación en nuestra pedagogía en el siglo XIX se destacan José Agustín Caballero (1762 - 1835), Félix Varela Morales (1788 - 1853), José de la Luz y Caballero (1800 - 1882), Rafael María Mendive (1821 - 1886), Rafael Morales (Moralitos) (1845 - 1877), Manuel Valdés Rodríguez (1849 - 1914), José Julián Martí Pérez (1853 - 1895), Enrique José Varona y Pera (1849-1933), entre otros, quienes plantearon que la formación institucionalizada debía preparar al estudiantado para que se insertara en la sociedad de manera ética y comprometida con su tiempo.

A la luz del pensamiento de Martí, Che y Fidel, educar es sembrar valores, inculcar y desarrollar sentimientos, transformar las criaturas que vienen al mundo con

58 Una mirada a los valores en el contexto universitario: Cuba Edelmis Cristina Reyes-Quiñones

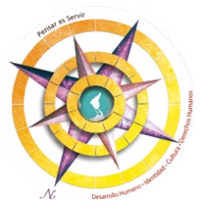


imperativos de la naturaleza, muchas veces contradictorios con las virtudes que más se aprecian, como la solidaridad, desprendimiento, valentía y fraternidad y otras ideas que constituyen paradigmas para la formación y fortalecimiento de los valores en la nueva generación.

Las mejores tradiciones educativas se sintetizan brillantemente en el pensamiento pedagógico del Che y Fidel, cuando señalan que "...la política educacional del Partido tiene como fin, formar a todo el pueblo en la concepción científica del mundo, desarrollar en toda su plenitud humana las capacidades intelectuales, físicas y espirituales del individuo y fomentar en él, elevados sentimientos y gustos estéticos; convertir los principios ideo-políticos y morales en convicciones personales y hábitos de conducta" (Chávez, 1996, p.18).

Los valores, han sido evaluados con profundidad en los últimos años por investigaciones como clarificación de valores: Caduto (1992), fortalecimiento de valores: Aguilar (1998); formación de valores: Fabelo (1989, 1995, 1996, 1998, 2003), González (1993, 1995, 1996, 1997, 1998), Rangel (1999), Chacón(1996,1999, 2002), Chávez (2000), Rodríguez (2000), Díaz (2001), Sáez (2001), Izquierdo (2002), Ojalvo (2002),
López (2002), Domínguez (2003), Aldea (2005), Arana (2005) y Ávalos (2006).

Los valores son determinaciones espirituales que designan la significación positiva de las cosas, hechos, fenómenos, relaciones y sujetos, para un individuo, un grupo, clase social o sociedad en su conjunto (VIII Seminario Nacional, 2008, p. 3). Por su contenido, los valores son orientadores, reguladores y autorreguladores de la conducta, constituyen un sistema pues guardan relación dinámica unos con otros y conforman una jerarquía entre ellos que es decisiva en los momentos de elección moral. Su educación es un proceso activo, complejo y contradictorio.

Trabajar los valores constituye un objetivo esencial de la educación superior cubana, son respaldados en los proyectos educativos en los que docentes ocupan un papel rector en el sistema de influencias que propician la formación y la enseñanza en la que asumen un papel cada vez más protagónico en la conducción de su aprendizaje (Bauzá Vázquez y Marañón Rodríguez, 2012).

Concluimos que se necesita la educación en valores para promover cambios significativos que conduzcan a la formación de un ser humano capaz de desenvolverse en la sociedad, 
que pueda practicar como norma de vida la libertad, la solidaridad, la honestidad, el respeto, la paz y la justicia social. Además, que contribuya a formar hombres y mujeres integrales capaces de dar respuesta a la crisis social y a la desvalorización humana que se vive actualmente.

\section{Referencias}

Aguilar Díaz, C. (1998). Fortalecimiento de valores: Una necesidad de todos los tiempos. Instituto Superior Politécnico José Martí: Cuba

Bauzá Vázquez, E. y Marañón Rodríguez, E. (Julio-septiembre, 2012). La formación y desarrollo de los valores en la educación superior cubana y su proceso de socialización. Revista Didáctica y Educación, 3(3), 89 -90.

Caduto, M. J. (1992). Guía para la enseñanza de valores ambientales. Los Libros de la Catarata. Serie de Educación Ambiental.

Castro Ruz, F. (10 de octubre de 1991). Discurso de inauguración del IV Congreso del Partido Comunista de Cuba, efectuado en el Teatro Heredia de Santiago de Cuba. Periódico Granma. Editora Política

Castro Ruz, F. (17 de noviembre de 2005). Discurso pronunciado en el Aula Magna de la Universidad de La Habana. Periódico Granma. Editora Política

CC PCC. (2012). Programa Director para la Educación en el Sistema de Valores de la Revolución Cubana. Material Digital.

CC PCC. (2017). Lineamientos de la Política Económica y Social del Partido y la Revolución. VII Congreso del Partido aprobados por el III Pleno del Comité Central el 18 de mayo y respaldados por la Asamblea Nacional del Poder Popular el 1 de junio de 2017.

CC PCC. 26 de octubre de 2006). Programa Director para el Reforzamiento de Valores fundamentales en la Sociedad Cubana actual. Material Digital.

CUJAE. (Julio del 2011). Estrategias y objetivos del Curso 2011-2012. Versión digital.

Díaz López, A. (2001). El potencial geográfico comunitario para la educación en valores desde la escuela. Comunidad Jayamá.

Díaz-Canel, M. (2010). Conferencia en el VII Congreso Internacional Universidad 2010. http://www.cubadebate.cu/especiales/2010/02/12.

Fabelo Corzo, J. R. (1999). Hacia una reconstrucción axiológica del socialismo, el mercado y los valores humanos. La Habana: Editorial José Martí

López Bombino, L. R. (2002). El diálogo y la cultura del error en la formación de valores. Editorial Felix Varela: La Habana

60 Una mirada a los valores en el contexto universitario: Cuba Edelmis Cristina Reyes-Quiñones 